Dieter Probst

\title{
On the relationship between fixed points and iteration in admissible set theory without foundation
}

Received: 1 November 2003 /

Published online: 27 December 2004 - (C) Springer-Verlag 2004

\begin{abstract}
In this article we show how to use the result in Jäger and Probst [7] to adapt the technique of pseudo-hierarchies and its use in Avigad [1] to subsystems of set theory without foundation. We prove that the theory $\mathrm{KPi}^{0}$ of admissible sets without foundation, extended by the principle ( $\Sigma$-FP), asserting the existence of fixed points of monotone $\Sigma$ operators, has the same proof-theoretic ordinal as $\mathrm{KPi}^{0}$ extended by the principle $(\Sigma-\mathrm{TR})$, that allows to iterate $\Sigma$ operations along ordinals. By Jäger and Probst [6] we conclude that the metapredicative Mahlo ordinal $\varphi \omega 00$ is also the ordinal of $\mathrm{KPi}^{0}+(\Sigma-\mathrm{FP})$. Hence the relationship between fixed points and iteration persists in the framework of set theory without foundation.
\end{abstract}

\section{Introduction}

In classical set theory, the relationship between fixed points and iteration is evident. Given a monotone operator $\Gamma$ on the power set $\mathrm{P}(a)$ of some set $a$, a fixed point is obtained by iterating the operator along the ordinals: Start with an arbitrary set $I_{\Gamma}^{0}:=x \subseteq a$, apply the operator in the successor case, i.e. $I_{\Gamma}^{\alpha+1}:=\Gamma\left(I_{\Gamma}^{\alpha}\right)$ and take the union at limit stages, i.e. $I_{\Gamma}^{\lambda}:=\bigcup_{\{\alpha<\lambda\}} I_{\Gamma}^{\alpha}$. The monotonicity of $\Gamma$ and a cardinality argument assure that there is an ordinal $\sigma$ such that $I_{\Gamma}^{\sigma}$ is a fixed point of the operator $\Gamma$. On the other hand, iterating an operator along a well-ordering can be seen as the iterative construction of a fixed point of a suitable monotone operator.

In (meta)-predicative subsystems of second order arithmetic things are more complex. One cannot prove the existence of well-orderings long enough for the iteration process to become stationary. Nevertheless, the existence of fixed points follows if we assume that the well-orderedness of $\prec$ implies the existence of a hierarchy $H$ with $(H)_{\alpha}=I_{\Gamma}^{\alpha}$ for all $\alpha$ in the field of $\prec$. This principle is called arithmetical transfinite recursion, or (ATR) for short, and was shown by Avigad [1] to be equivalent over the theory $\mathrm{ACA}_{0}$ to the principle (FP) that claims the existence of fixed points of monotone operators defined by an arithmetical formula. To prove the existence of fixed points given (ATR), Avigad makes use of so called

D. Probst: Institut für Informatik und angewandte Mathematik, Universität Bern, Neubrückstrasse 10, 3012 Bern, Switzerland

The author is supported by the Swiss National Science Foundation.

Key words or phrases: Admissible sets - Fixed points - Iteration - Pseudo-hierarchies 
pseudo-hierarchies, i.e. hierarchies where the underlying ordering is only a linear ordering, not a well-ordering. Their existence follows from the fact that over $\mathrm{ACA}_{0}$ the $\Pi_{1}^{1}$ formula $W O(\prec)$, asserting that $\prec$ is a well-ordering, is not equivalent to any $\Sigma_{1}^{1}$ formula. For the other direction, Avigad showed that a hierarchy can be obtained as a fixed point of a suitable monotone operator describing the inductive build-up of the hierarchy.

Our aim is to use pseudo-hierarchies also in the framework of Kripke-Platek set theory without foundation. The problem is, that it is now consistent to assume that there is a $\Sigma$ formula $A(\prec)$ equivalent to the statement " $\prec$ is a well-ordering". Under this assumption, the existence of pseudo-hierarchies is in general not provable. Moreover, "being a well-ordering" is then a $\Delta$ predicate. Hence $\Pi_{1}^{1}$ comprehesion is available, and in particular, the ordinal of the theory $\mathrm{KPi}^{0}+(\Sigma-\mathrm{TR})$, the metapredicative Mahlo ordinal $\varphi \omega 00$, becomes provable. To save the pseudohierarchy argument, we observe that the theory $\mathrm{KPi}^{0}+(\Sigma-\mathrm{TR})$ can be consistently extended by an axiom claiming the existence of a set for which transfinite induction up to $\varphi \omega 00$ fails. As follows from Jäger and Probst [7], the resulting theory $\left[\mathrm{KPi}^{0}+(\Sigma-\mathrm{TR})\right]^{\dagger}$ still has the same proof-theoretic ordinal as $\mathrm{KPi}^{0}+(\Sigma-\mathrm{TR})$, but being a well-ordering is no longer a $\Delta$ predicate. Consequently, the technique of pseudo-hierarchies can be applied to prove the existence of fixed points of monotone $\Sigma$ operators, given the iteration principle $(\Sigma$-TR). Hence $\varphi \omega 00$ is an upper bound for $\mathrm{KPi}^{0}+(\Sigma-\mathrm{FP})$. To show that $\varphi \omega 00$ is also a lower bound, we argue how the well-ordering proof for the theory $\mathrm{KPi}^{0}+(\Sigma$-TR) given in Jäger and Probst [6] can be adjusted to the theory $\mathrm{KPi}^{0}+(\Sigma-\mathrm{FP})$. This concludes the argument that extending $\mathrm{KPi}^{0}$ either by an axiom that allows us to iterate $\Sigma$ operations along arbitrary well-orderings or an axiom that asserts the existence of fixed points of monotone $\Sigma$ operators leads to theories of the same proof-theoretic strength. It that sense the relationship between fixed points and iteration persists in the framework of admissible set theory without foundation.

The plan of this paper is as follows: In the next section we introduce the theories $\mathrm{KPi}^{0}+(\Sigma-\mathrm{FP})$ and $\mathrm{KPi}^{0}+(\Sigma-\mathrm{TR})$. Section 3 recalls some ordinal theoretic facts that are relevant in the sequel. In section 4 we show how $\left[\mathrm{KPi}^{0}+(\Sigma-\mathrm{TR})\right]^{\dagger}$ proves the existence of fixed points of monotone $\Sigma$ operators on the power set of the natural numbers. This gives us $\varphi \omega 00$ as an upper bound. That $\varphi \omega 00$ is also a lower bound is shown in section 5 , and section 6 contains some concluding remarks.

\section{The theories $\mathrm{KPi}^{0}+(\Sigma-\mathrm{FP})$ and $\mathrm{KPi}^{0}+(\Sigma-\mathrm{TR})$}

We start by giving a short review of the theory $\mathrm{KPi}^{0}$. Our version of $\mathrm{KPi}^{0}$ is formulated with the natural numbers as urelements. Accordingly, we let $L_{1}$ denote a standard languages of first order arithmetic with variables $a, b, c, \ldots, u, v, w$, $x, y, z, \ldots$ (possibly with subscripts), a constant 0 as well as function and relation symbols for all primitive recursive functions and relations. The theory $\mathrm{KPi}^{0}$ is formulated in the extension $\mathcal{L}^{*}=L_{1}(\in, N, S, A d)$ of $L_{1}$ by the membership relation symbol $\in$, the set constant $\mathrm{N}$ for the set of natural numbers and the unary relation symbols $\mathrm{S}$ and Ad for sets and admissible sets, respectively. 
The number terms of $\mathcal{L}^{*}$ are inductively generated from the variables, the constant 0 and the symbols for the primitive recursive functions; the terms $r, s, t, \ldots$ of $\mathcal{L}^{*}$ are the number terms of $\mathrm{L}_{1}$ plus the set constant $\mathrm{N}$. The formulas $A, B, C, \ldots$ of $\mathcal{L}^{*}$ as well as the $\Delta_{0}, \Sigma, \Pi, \Sigma_{n}$ and $\Pi_{n}$ formulas of $L_{1}$ are defined as usual. Further, equality between objects is not represented by a primitive symbol, but defined by

$$
(s=t):=\left\{\begin{array}{l}
(s \in \mathrm{N} \wedge t \in \mathrm{N} \wedge(s=\mathrm{N} t)) \vee \\
(\mathrm{S}(s) \wedge \mathrm{S}(t) \wedge(\forall x \in s)(x \in t) \wedge(\forall x \in t)(x \in s))
\end{array}\right.
$$

where $=\mathrm{N}$ is the symbol for the primitive recursive equality on the natural numbers. The formula $A^{s}$ is the result of replacing each unrestricted quantifier $\exists x(\ldots)$ and $\forall x(\ldots)$ in $A$ by $(\exists x \in s)(\ldots)$ and $(\forall x \in s)(\ldots)$, respectively. In addition, we freely make use of all standard set-theoretic notations. So we write Fun $(f)$ and $\operatorname{Tran}(x)$ for the $\Delta_{0}$ formulas expressing that $f$ is a function and that $x$ is a transitive set. Field $(x)$ is used to denote the field of an ordering $x$ and Dom $(f)$ denote the set $\{u: \exists v(u, v) \in f\}$, and $\operatorname{Rng}(f)$ denotes the set $\{v: \exists u(u, v) \in f\}$, where $(x, y)$ stands for the standard ordered pair, $\{\{x\},\{x, y\}\}$. As usual, $x \subseteq y$ states that $x$ is a subset of the set $y$, i.e. $x \subseteq y:=\mathrm{S}(x) \wedge \mathrm{S}(y) \wedge(\forall u \in x)(u \in y)$.

The $\mathcal{L}^{*}$ theory $\mathrm{KPi}^{0}$ is based on classical first order logic with equality. Its non-logical axioms can be divided into the following five groups.

I. Ontological axioms. We have for all terms $r$, $\mathbf{s}$ and $t$ of $\mathrm{L}_{1}$, all function symbols $\mathcal{H}$ and relation symbols $\mathcal{R}$ of $\mathrm{L}_{1}$ and all axioms $A(\mathbf{a})$ of group III whose free variables belong to the list $\mathbf{a}$ :

(1) $a \in \mathrm{N} \leftrightarrow \neg \mathrm{S}(a)$,

(2) $\mathbf{a} \in \mathrm{N} \rightarrow \mathcal{H}(\mathbf{a}) \in \mathrm{N}$,

(3) $\mathcal{R}(\mathbf{a}) \rightarrow \mathbf{a} \in \mathrm{N}$,

(4) $a \in b \rightarrow \mathrm{S}(b)$,

(5) $\operatorname{Ad}(a) \rightarrow(\mathrm{N} \in a \wedge \operatorname{Tran}(a))$,

(6) $\operatorname{Ad}(a) \rightarrow(\forall \mathbf{x} \in a) A^{a}(\mathbf{x})$,

(7) $\operatorname{Ad}(a) \wedge \operatorname{Ad}(b) \rightarrow a \in b \vee a=b \vee b \in a$.

II. Number-theoretic axioms. We have for all axioms $A(\mathbf{a})$ of Peano arithmetic PA which are not instances of the schema of complete induction and whose free variables belong to the list $\mathbf{a}$ :

(Number theory) $\mathbf{a} \in \mathrm{N} \rightarrow A^{\mathrm{N}}(\mathbf{a})$.

III. Kripke Platek axioms. For all $\Delta_{0}$ formulas $A(u)$ and $B(u, v)$ of $\mathcal{L}^{*}$ :

(Pair) $\exists x(a \in x \wedge b \in x)$,

(Tran) $\exists x(a \subseteq x \wedge \operatorname{Tran}(x))$,

$\left(\Delta_{0}\right.$-Sep) $\exists y(\mathrm{~S}(y) \wedge y=\{x \in a: A(x)\})$,

$\left(\Delta_{0}\right.$-Col) $(\forall x \in a) \exists y B(x, y) \rightarrow \exists z(\forall x \in a)(\exists y \in z) B(x, y)$.

IV. Limit axiom. It is used to formalize that each set is element of an admissible set, hence we claim:

(Lim) $\exists x(a \in x \wedge \operatorname{Ad}(x))$. 
V. Complete induction on $\mathrm{N}$. The only induction principle included in the axioms of $\mathrm{KPi}^{0}$ is the following axiom of complete induction on the natural numbers for sets:

$\left(\mathrm{S}-\mathrm{I}_{\mathrm{N}}\right) 0 \in a \wedge(\forall x \in \mathrm{N})(x \in a \rightarrow x+1 \in a) \rightarrow \mathrm{N} \subseteq a$.

The monograph Barwise [2] provides an excellent introduction into general admissible set theory. Theories of admissible sets without foundation, on the other hand, have been studied, in particular, in Jäger [3, 4]. It is shown there, among other things, that the proof-theoretic ordinal of $\mathrm{KPi}^{0}$ is $\Gamma_{0}$.

In this article, however, we are mainly interested in the relationship between the theory $\mathrm{KPi}^{0}+(\Sigma-\mathrm{FP})$ that extends $\mathrm{KPi}^{0}$ by the axiom schema $(\Sigma-\mathrm{FP})$, claiming the existence of fixed points of monotone $\Sigma$ operators on the power set of the natural numbers, and the theory $\mathrm{KPi}^{0}+(\Sigma$-TR), allowing to iterate $\Sigma$ operators along ordinals. In order to give a compact formulation of our principle $(\Sigma-\mathrm{FP})$ we introduce the following abbreviations: For all formulas $A(\mathbf{u}, v, w)$ of $\mathcal{L}^{*}$ we set

$$
\begin{aligned}
\mathrm{Op}_{A}^{\mathrm{N}}(\mathbf{a}) & :=(\forall x \subseteq \mathrm{N})(\exists ! y \subseteq \mathrm{N}) A(\mathbf{a}, x, y), \\
\operatorname{Mon}_{A}^{\mathrm{N}}(\mathbf{a}) & :=(\forall u, v, x, y \subseteq \mathrm{N})[A(\mathbf{a}, u, x) \wedge A(\mathbf{a}, v, y) \wedge u \subseteq v \rightarrow x \subseteq y] .
\end{aligned}
$$

$\mathrm{Op}_{A}^{\mathrm{N}}(\mathbf{a})$ expresses that for the parameters a the formula $A(\mathbf{a}, x, y)$ defines a functional relation on $\mathrm{P}(\mathrm{N})$. If in addition $\operatorname{Mon}_{A}^{N}(\mathbf{a})$ holds, this functional relation is monotone in the usual sense and we say that $A$ defines a monotone operator for the parameters a. The principle ( $\Sigma$-FP) takes now the following form: For each $\Sigma$ formula $A(\mathbf{u}, v, w)$ of $\mathcal{L}^{*}$ with at most the variables $\mathbf{u}, v, w$ free we have

$$
\mathrm{Op}_{A}^{\mathrm{N}}(\mathbf{a}) \wedge \operatorname{Mon}_{A}^{\mathrm{N}}(\mathbf{a}) \rightarrow(\exists x \subseteq \mathrm{N}) A(\mathbf{a}, x, x) .
$$

Remark 1. The purpose of the restriction to operators on $\mathrm{P}(\mathrm{N})$ is to keep the fixed point axiom persistent. If instead, we add to $\mathrm{KPi}^{0}$ a fixed point principle that claims arbitrary monotone $\Sigma$ operators to have fixed points, we do not increase the prooftheoretic strength. In this case however, a further strengthening, e.g. by foundation leads to an inconsistent theory. For a further discussion, see section 6.

The theory $\mathrm{KPi}^{0}+(\Sigma-\mathrm{TR})$ is the extension of the theory $\mathrm{KPi}^{0}$ by the axiom schema ( $\Sigma$-TR) that allows to iterate $\Sigma$ operations along ordinals. In the current context, ordinals are hereditarily transitive well-founded sets:

$$
\begin{aligned}
\operatorname{Wf}(a, \in) & :=\forall x(x \subseteq a \wedge x \neq \emptyset \rightarrow(\exists y \in x)(\forall z \in y)(z \notin x)), \\
\operatorname{Ord}(a) & :=\operatorname{Tran}(a) \wedge(\forall x \in a) \operatorname{Tran}(x) \wedge \operatorname{Wf}(a, \in) .
\end{aligned}
$$

To formulate the iteration principle, we introduce for each $\Sigma$ formula $D(\mathbf{u}, x, y, z)$ of $\mathcal{L}^{*}$, with at most the variables $\mathbf{u}, x, y, z$ free, the formula

$$
\operatorname{Hier}_{D}^{\mathbb{Q}}(\mathbf{a}, b, f):=\left\{\begin{array}{l}
\operatorname{Ord}(b) \wedge \operatorname{Fun}(f) \wedge \operatorname{Dom}(f)=b \wedge \\
(\forall x \in b) D(\mathbf{a}, x, f\lceil x, f(x)) .
\end{array}\right.
$$

The iteration principle now takes the form

$$
\operatorname{Ord}(b) \wedge(\forall x \in b) \forall y \exists ! z D(\mathbf{a}, x, y, z) \rightarrow \exists f \operatorname{Hier}_{D}^{\mathbb{Q}}(\mathbf{a}, b, f)
$$

for all $\Sigma$ formulas $D(\mathbf{u}, x, y, z)$ of $\mathcal{L}^{*}$ with at most the variables $\mathbf{u}, x, y, z$ free. 
As shown in Jäger and Probst [6] the theory $\mathrm{KPi}^{0}+(\Sigma$-TR) enables us also to iterate $\Sigma$ operations along arbitrary well-orderings. Given a set $a$ and a binary relation $b \subseteq a \times a$, we write $\operatorname{Lin}(a, b)$ if $b$ is a strict linear ordering on $a$. A linear ordering is a well-ordering if any non-empty subset of its domain has a least element with respect to this ordering,

$$
\operatorname{Wo}(a, b):=\operatorname{Lin}(a, b) \wedge \forall x(x \subseteq a \wedge x \neq \emptyset \rightarrow(\exists y \in x)(\forall z \in x)((z, y) \notin b))
$$

We define for each $\Sigma$ formula $D(\mathbf{u}, x, y, z)$ of $\mathcal{L}^{*}$ the formula

$$
\operatorname{Hier}_{D}(\mathbf{a}, b, c, f):=\left\{\begin{array}{l}
\operatorname{Wo}(b, c) \wedge \operatorname{Fun}(f) \wedge \operatorname{Dom}(f)=b \wedge \\
(\forall x \in b) D(\mathbf{a}, x,\{(y, f(y)):(y, x) \in c\}, f(x))
\end{array}\right.
$$

in order to state the theorem below.

Theorem 1. For all $\Sigma$ formulas $D(\mathbf{u}, x, y, z)$ of $\mathcal{L}^{*}$ that contain at most the variables $\mathbf{u}, x, y, z$ free, $\mathrm{KPi}^{0}+(\Sigma$-TR $)$ proves

$$
\operatorname{Wo}(b, c) \wedge(\forall x \in b) \forall y \exists ! z D(\mathbf{a}, x, y, z) \rightarrow \exists f \operatorname{Hier}_{D}(\mathbf{a}, b, c, f) .
$$

In [6] it is also shown that the proof-theoretic ordinal of $\mathrm{KPi}^{0}+(\Sigma-\mathrm{TR})$ is $\varphi \omega 00$. In the sequel we will show that the theory $\mathrm{KPi}^{0}+(\Sigma-\mathrm{FP})$ has the same prooftheoretic ordinal, and hence unfolds yet another aspect of metapredicative Mahlo. The Mahlo axiom schema for admissible set theory without foundation claims $\Pi_{2}$ reflection on admissible sets,

$$
A(\mathbf{a}) \rightarrow \exists x\left(\mathbf{a} \in x \wedge \operatorname{Ad}(x) \wedge A^{x}(\mathbf{a})\right) \quad\left(\Pi_{2}-\operatorname{Ref}^{A d}\right)
$$

for all $\Pi_{2}$ formulas $A(\mathbf{u})$ of $\mathcal{L}^{*}$ with at most the variables $\mathbf{u}$ free. The theory $\mathrm{KPi}^{0}+\left(\Pi_{2}-\operatorname{Ref}^{A d}\right)$ is denoted by $\mathrm{KPm}^{0}$ and is analyzed in detail in Jäger and Strahm [8] and Strahm [13].

We conclude the section by mentioning a result about $\mathrm{KPi}^{0}$ that plays an important role in section 5 where we derive a lower bound for $\mathrm{KPi}^{0}+(\Sigma-\mathrm{FP})$. This result is due to Jäger and a detailed proof is given e.g. in [6].

Lemma 1. Let $D(\mathbf{u}, v)$ be a $\Delta_{0}$ formula of $\mathcal{L}^{*}$ with at most the variables $\mathbf{u}, v$ free. Then $\mathrm{KPi}^{0}$ proves that

$$
\begin{aligned}
& \exists x(D(\mathbf{a}, x) \wedge \mathbf{a} \in x \wedge \operatorname{Ad}(x)) \rightarrow \\
& \exists y(y=\bigcap\{x: D(\mathbf{a}, x) \wedge \mathbf{a} \in x \wedge \operatorname{Ad}(x)\} \wedge D(\mathbf{a}, y) \wedge \mathbf{a} \in y \wedge \operatorname{Ad}(y)) .
\end{aligned}
$$

This lemma implies for example that in $\mathrm{KPi}^{0}$ for any set $x$ the intersection $x^{+}$of all admissibles containing $x$ is an admissible itself,

$$
x^{+}:=\bigcap\{y: x \in y \wedge \operatorname{Ad}(y)\}
$$




\section{Ordinal-theoretic preliminaries}

The aim of this section is to review some ordinal-theoretic facts that will be relevant in the sequel. As in, for example, Jäger and Strahm [8] we work with the ternary Veblen function, that is obtained from the binary $\varphi$ function as follows:

(1) $\varphi 0 \beta \gamma$ is $\varphi \beta \gamma$,

(2) if $\alpha>0$, then $\varphi \alpha 0 \gamma$ denotes the $\gamma$ th ordinal which is strongly critical with respect to all functions $\lambda \xi . \lambda \eta . \varphi \delta \xi \eta$ for $\delta<\alpha$,

(3) if $\alpha>0$ and $\beta>0$, then $\varphi \alpha \beta \gamma$ denotes the $\gamma$ th common fixed point of the functions $\lambda \eta . \varphi \alpha \delta \eta$ for $\delta<\eta$.

Let $\Xi_{0}$ be the least ordinal greater than 0 which is closed under addition and the ternary $\varphi$ function. In the following we will work with a standard primitive recursive notation system $(\mathrm{OT}, \triangleleft)$ for all ordinals less than $\Xi_{0}$. All required definitions are straightforward generalizations of those used for building a notation system for $\Gamma_{0}$ (cf. $[9,11]$ ) and are omitted. In the reminder of this article we let $\mathfrak{a}, \mathfrak{b}, \mathfrak{c}, \ldots$ range other the set OT and the terms $\hat{0}, \hat{1}, \hat{2}, \ldots$ act as codes for the finite ordinals. Moreover, ordinal constants such as $0,1, \omega$ and $\varphi \omega 00$ are often used instead of the corresponding ordinal notations.

We set for all primitive recursive relations $\prec$ and all number terms $s$ :

$$
\begin{aligned}
\operatorname{Prog}(\prec, x) & :=(\forall u \in \operatorname{Field}(\prec))[\forall v(v \prec u \rightarrow v \in x) \rightarrow u \in x], \\
\operatorname{TI}(\prec, x) & :=\operatorname{Prog}(\prec, x) \rightarrow \operatorname{Field}(\prec) \subseteq x . \\
\operatorname{TI}(\prec, x, s) & :=\operatorname{Prog}(\prec, x) \rightarrow(\forall n \prec s)(n \in x) .
\end{aligned}
$$

To be consistent with the notation in [6] we often write $\operatorname{TI}(\mathfrak{a}, x)$ instead of $\operatorname{TI}(\triangleleft, \mathfrak{a}, x)$, where $\triangleleft$ is the special well-ordering of our notation system. An ordinal $\alpha$ is called provable in the theory $\mathrm{T}$ - formulated in $\mathcal{L}^{*}$ or a similar language - if there exists a primitive recursive well-ordering $\prec$ of order-type $\alpha$ such that

$$
\mathrm{T} \vdash \forall x \operatorname{TI}(\prec, x) .
$$

The least ordinal which is not provable in T is called the proof-theoretic ordinal of $\mathrm{T}$ and denoted by $|\mathrm{T}|$.

\section{Upper bound}

To determine an upper bound for the theory $\mathrm{KPi}^{0}+(\Sigma-\mathrm{FP})$, we show that the fixed point axiom $(\Sigma$-FP $)$ is provable in a theory denoted by $\left[\mathrm{KPi}^{0}+(\Sigma-\mathrm{TR})\right]^{\dagger}$ which is a conservative extension of $\mathrm{KPi}^{0}+(\Sigma$-TR $)$ in the sense that its proof-theoretic ordinal is still $\varphi \omega 00$. The theory $\left[\mathrm{KPi}^{0}+(\Sigma-\mathrm{TR})\right]^{\dagger}$ is designed to prove the existence of so called pseudo-hierarchies. This allows us to adapt Avigad's argument in [1] to show that $\left[\mathrm{KPi}^{0}+(\Sigma-\mathrm{TR})\right]^{\dagger}$ proves the existence of fixed points of monotone $\Sigma$ operators.

As shown in [6], $\mathrm{KPi}^{0}+(\Sigma$-TR $)$ has proof-theoretic ordinal $\varphi \omega 00$. Therefore the sentence

$$
\forall x \operatorname{TI}(x, \varphi \omega 00)
$$


is not provable in $\mathrm{KPi}^{0}+(\Sigma-\mathrm{TR})$ and hence the theory $\left[\mathrm{KPi}^{0}+(\Sigma-\mathrm{TR})\right]^{\dagger}$ that extends the theory $\mathrm{KPi}^{0}+(\Sigma-\mathrm{TR})$ by the axiom

$$
\exists x \neg \operatorname{TI}(x, \varphi \omega 00)
$$

is consistent. Moreover, this extension still has the same proof-theoretic ordinal.

\section{Lemma 2.}

$$
\left|\mathrm{KPi}^{0}+(\Sigma-\mathrm{TR})\right|=\left|\left[\mathrm{KPi}^{0}+(\Sigma-\mathrm{TR})\right]^{\dagger}\right| .
$$

Proof. This result is a consequence of results in Jäger and Probst [7]. For the reader's convenience we summarize the main steps. Assume that there is a primitive recursive well-ordering $\prec$ such that

$$
\left[\mathrm{KPi}^{0}+(\Sigma-\mathrm{TR})\right]^{\dagger} \vdash \forall x \mathrm{TI}(\prec, x) .
$$

Our aim is to show that the ordertype of $\prec$ is less than $\varphi \omega 00$. Since $\mathrm{KPm}^{0}$ proves the iteration principle ( $\Sigma$-TR) (cf. [6]) we have that

$$
\mathrm{KPm}^{0} \vdash \exists x \neg \operatorname{TI}(x, \varphi \omega 00) \rightarrow \forall y \operatorname{TI}(\prec, y) .
$$

It follows from the proof-theoretic analysis of $\mathrm{KPm}^{0}$ given in Jäger and Strahm [8] that a standard semi-formal system $\mathrm{PA}^{\infty}$ for second order arithmetic proves

$$
\mathrm{PA}^{\infty} \frac{\alpha_{0}}{0} \operatorname{TI}(\varphi \omega 00, X), \operatorname{TI}(\prec, Y)
$$

for an $\alpha_{0}<\varphi \omega 00$.

Inspecting the proof of the boundedness lemma (cf. e.g. Pohlers [9]) we observe that also the following slightly more general statement holds:

Lemma 3 (Boundedness Lemma). Suppose that $\prec_{1}$ and $\prec_{2}$ are primitive recursive well-orderings, $\Gamma$ is a finite set of $U$-positive formulas of $\mathrm{PA}^{\infty}, \Delta$ is a finite set of $V$-positive formulas of $\mathrm{PA}^{\infty}, V$ does not occur in $\Gamma$ and $U$ does not occur in $\Delta$. Further, let $\Lambda_{\mathbf{S}}:=\left\{s_{1} \notin U, \ldots, s_{k} \notin U\right\}$ and $\Lambda_{\mathbf{t}}:=\left\{t_{1} \notin V, \ldots, t_{l} \notin V\right\}$. If

$$
\mathrm{PA}^{\infty} \mid \frac{\alpha}{0} \neg \operatorname{Prog}\left(\prec_{1}, U\right), \Lambda_{\mathbf{s}}, \Gamma, \neg \operatorname{Prog}\left(\prec_{2}, V\right), \Lambda_{\mathbf{t}}, \Delta
$$

then it follows that

$$
\mathbb{N} \models \bigvee\left\{F\left[\left(\prec_{1}\right)_{\gamma_{1}} / U\right]: F \in \Gamma\right\} \quad \text { or } \quad \mathbb{N} \models \bigvee\left\{F\left[\left(\prec_{2}\right)_{\gamma_{2}} / V\right]: F \in \Delta\right\},
$$

where we have $\gamma_{i}=\beta_{i}+2^{\alpha}$ and $\beta_{1}=\max \left\{\left|s_{1}^{\mathbb{N}}\right|_{\prec_{1}}, \ldots,\left|s_{k}^{\mathbb{N}}\right|_{\prec_{1}}\right\}$ and $\beta_{2}=$ $\max \left\{\left|t_{1}^{\mathbb{N}}\right|_{\prec_{2}}, \ldots,\left|t_{l}^{\mathbb{N}}\right|_{\prec_{2}}\right\}$.

As in [9], if $\prec$ is a well-ordering we assign to each natural number in the field of $\prec$ an ordinal by the recursion $|n|_{\prec}:=\left\{|m|_{\prec}: m \prec n\right\}$, and $(\prec)_{\sigma}$ is the set $\left\{n \in \mathbb{N}:|n|_{\prec} \in \sigma\right\}$. We write $t^{\mathbb{N}}$ for the value of the closed term $t$ and $\mathbb{N} \models F[S / U]$ to indicate that $F$ holds in the standard model provided the relation symbol $U$ is interpreted by the set $S$.

Applying the above lemma to $(*)$ yields that

$$
\mathbb{N}=\forall x \in \operatorname{Field}(\varphi \omega 00)\left(|x|_{\triangleleft} \leq \omega^{\alpha_{0}}\right) \text { or } \mathbb{N} \models \forall x \in \operatorname{Field}(\prec)\left(|x|_{\prec} \leq \omega^{\alpha_{0}}\right) \text {. }
$$

The first statement is obviously wrong and hence the ordertype of $\prec$ is less than $\varphi \omega 00$. 
Pseudo-hierarchies are hierarchies where the underlying ordering is not a wellordering. If $D(\mathbf{u}, x, y, z)$ is a formula of $\mathcal{L}^{*}$ we write

$$
\mathrm{PSH}_{D}(\mathbf{a}, b, c, f):=\left\{\begin{array}{l}
\operatorname{Lin}(b, c) \wedge \neg \operatorname{Wo}(b, c) \wedge \operatorname{Fun}(f) \wedge \operatorname{Dom}(f)=b \wedge \\
(\forall x \in b) D(\mathbf{a}, x,\{(y, f(y)):(y, x) \in c\}, f(x))
\end{array}\right.
$$

to express that $f$ is a pseudo-hierarchy for $D(\mathbf{u}, x, y, z)$ with parameters a along the ordering $c$ on $b$.

The next lemma is a key step in showing that $\left[\mathrm{KPi}^{0}+(\Sigma-\mathrm{TR})\right]^{\dagger}$ proves the existence of pseudo-hierarchies for $\Sigma$ formulas of $\mathcal{L}^{*}$. It states that there is no $\Sigma$ formula $A(u, v)$ that is equivalent to the assertion that $b$ is a well-ordering. The proof of the lemma exhibits the role of the axiom $\exists x \neg \operatorname{TI}(\triangleleft, x, \varphi \omega 00)$ : It is to exclude that $\operatorname{Wo}(x, y)$ is a $\Delta$ predicate.

Lemma 4. For all $\Sigma$ formulas $A(\mathbf{u}, v, w)$ of $\mathcal{L}^{*}$ the theory $\left[\mathrm{KPi}^{0}+(\Sigma-\mathrm{TR})\right]^{\dagger}$ proves:

$$
\forall \mathbf{x} \neg(\forall y, z[A(\mathbf{x}, y, z) \leftrightarrow \operatorname{Wo}(y, z)]) .
$$

Proof. Assume that there are sets a and a $\Sigma$ formula $A(\mathbf{u}, v, w)$ such that

$$
\forall y, z[A(\mathbf{a}, y, z) \leftrightarrow \operatorname{Wo}(y, z)]
$$

holds. We show that under this assumption we could embed $\Pi_{1}^{1}-\mathrm{CA}_{0}$ into the theory $\left[\mathrm{KPi}^{0}+(\Sigma-\mathrm{TR})\right]^{\dagger}$. Thus the theory $\left[\mathrm{KPi}^{0}+(\Sigma-\mathrm{TR})\right]^{\dagger}$ would prove $(\forall x \subseteq$ $\mathrm{N}) \operatorname{TI}(x, \varphi \omega 00)$. Since our ordinal notations are elements of $\mathrm{N}$ and $\Pi_{1}^{1}-\mathrm{CA}_{0}$ proves the ordinal $\varphi \omega 00$, this contradicts $\exists x \neg \mathrm{TI}(x, \varphi \omega 00)$.

Let $\Psi(u, \mathbf{v}, \mathbf{V})$ be a $\Pi_{1}^{1}$ formula of $L_{2}$. As shown e.g. in [12], ACA proves that for all natural numbers $\mathbf{m}$ and all subsets $\mathbf{Y}$ of $\mathbf{N}$ there is a set $T$ dependent on $\Psi$ and the parameters $\mathbf{m}$ and $\mathbf{Y}$ such that for all $x$,

(1) $(T)_{x}$ is a tree,

(2) $(T)_{x}$ is recursive in $\mathbf{Y}$,

(3) $\Psi(x, \mathbf{m}, \mathbf{Y}) \leftrightarrow \mathrm{WO}\left(\mathrm{KB}\left((T)_{x}\right)\right)$,

where $\mathrm{KB}(S)$ denotes the restriction of the Kleene/Brower ordering to the tree $S$, $\mathrm{WO}(X)$ is the $\mathrm{L}_{2}$ formula saying that $\{(x, y):\langle x, y\rangle \in X\}$ is a linear ordering that is well-founded and $(X)_{t}:=\{x:\langle x, t\rangle \in X\}$, where $\langle\cdot, \cdot\rangle$ is a standard primitive recursive pairing function. To simplify the notation we also use capital letters $X, Y, Z, \ldots$ in $\mathcal{L}^{*}$ formulas to denote subsets of $\mathrm{N}$.

If we write $\mathrm{Wo}(z)$ for $\mathrm{Wo}(\operatorname{Field}(z), z)$ and let $\Psi^{*}$ denote the translation of $\Psi$ to the language $\mathcal{L}^{*}$ then $\left[\mathrm{KPi}^{0}+(\Sigma-\mathrm{TR})\right]^{\dagger}$ proves that

$$
(\forall x \in \mathrm{N})\left[\Psi^{*}(x, \mathbf{m}, \mathbf{Y}) \leftrightarrow \mathrm{Wo}\left(\left\{(u, v):\langle u, v\rangle \in \mathrm{KB}\left((T)_{x}\right)\right\}\right)\right] .
$$

Now the assumption $(*)$ puts us in position to apply $\Delta$ separation, i.e. there is a set $\mathrm{Z} \subseteq \mathrm{N}$ with

$$
\forall x\left[x \in Z \leftrightarrow x \in \mathrm{N} \wedge \Psi^{*}(x, \mathbf{m}, \mathbf{Y})\right] .
$$

But this means that we could embed $\Pi_{1}^{1}-\mathrm{CA}_{0}$ into $\left[\mathrm{KPi}^{0}+(\Sigma-\mathrm{TR})\right]^{\dagger}$. 
The ordering underlying a hierarchy is by definition a well-ordering. This fact allows us to prove properties of the hierarchy by transfinite induction along this well-ordering. For pseudo-hierarchies we can not apply this method of proof. Fortunately this does not matter, since $\left[\mathrm{KPi}^{0}+(\Sigma-\mathrm{TR})\right]^{\dagger}$ proves the existence of pseudo-hierarchies with "nice" properties:

Lemma 5 (Existence of pseudo-hierarchies). Assume that $D(\mathbf{u}, x, y, z)$ and $B(\mathbf{u}, x, y, z)$ are $\Sigma$ formulas of $\mathcal{L}^{*}$ and let $A$ be an arbitrary formula of $\mathcal{L}^{*}$ such that $\left[\mathrm{KPi}^{0}+(\Sigma-\mathrm{TR})\right]^{\dagger}$ proves

$$
A \rightarrow \forall b, c\left[\mathrm{Wo}(b, c) \rightarrow \exists f\left(\operatorname{Hier}_{D}(\mathbf{a}, b, c, f) \wedge B(\mathbf{a}, b, c, f)\right)\right] .
$$

Then $\left[\mathrm{KPi}^{0}+(\Sigma-\mathrm{TR})\right]^{\dagger}$ also proves

$$
A \rightarrow \exists d, e, g\left[\mathrm{PSH}_{D}(\mathbf{a}, d, e, g) \wedge B(\mathbf{a}, d, e, g)\right] .
$$

Proof. Let $A$ and a such that $\left[\mathrm{KPi}^{0}+(\Sigma-\mathrm{TR})\right]^{\dagger}$ proves

$$
A \rightarrow \forall b, c\left[\mathrm{Wo}(b, c) \rightarrow \exists f\left(\operatorname{Hier}_{D}(\mathbf{a}, b, c, f) \wedge B(\mathbf{a}, b, c, f)\right)\right] .
$$

Now we define

$$
\begin{aligned}
\operatorname{Hier}_{D}^{\prime}(\mathbf{a}, b, c, f): & =\left\{\begin{array}{l}
\operatorname{Lin}(b, c) \wedge \operatorname{Fun}(f) \wedge \operatorname{Dom}(f)=b \wedge \\
(\forall x \in b) D(\mathbf{a}, x,\{(y, f(y)):(y, x) \in c\}, f(x))
\end{array}\right. \\
E(\mathbf{a}, b, c): & =\exists f\left[\operatorname{Hier}_{D}^{\prime}(\mathbf{a}, b, c, f) \wedge B(\mathbf{a}, b, c, f)\right] .
\end{aligned}
$$

Note that $E(\mathbf{a}, b, c)$ is a $\Sigma$ formula of $\mathcal{L}^{*}$. Hence lemma 4 yields that

$$
\neg \forall x, y[E(\mathbf{a}, x, y) \leftrightarrow \mathrm{Wo}(x, y)] .
$$

So there are sets $d$ and $e$ such that

$$
[\neg \mathrm{Wo}(d, e) \wedge E(\mathbf{a}, d, e)] \vee[\operatorname{Wo}(d, e) \wedge \neg E(\mathbf{a}, d, e)] .
$$

Assuming $A$, the second disjunct contradicts (*), and the first disjunct is equivalent to

$$
\exists g\left[\mathrm{PSH}_{D}(\mathbf{a}, d, e, g) \wedge B(\mathbf{a}, d, e, g)\right] .
$$

To prove the existence of a fixed point of a given monotone $\Sigma$ operation on the powerset of $\mathrm{N}$, we first show that there is a pseudo-hierarchy such that the $\Sigma$ operation specifies the transition from one stage of the hierarchy to the next. Then this pseudo-hierarchy is divided into an upper part that contains no least stage, and the corresponding lower part. The careful definition of the pseudo-hierarchy then implies that the union of the stages in the lower part equals the intersection of the stages in the upper part and is also a fixed point of the given operation. The details of this argument are carried out in the proof of the following theorem. 
Theorem 2 (Existence of fixed points). Let $F(\mathbf{u}, x, y)$ be a $\Sigma$ formula of $\mathcal{L}^{*}$. Then $\left[\mathrm{KPi}^{0}+(\Sigma-\mathrm{TR})\right]^{\dagger}$ proves

$$
\mathrm{Op}_{F}^{\mathrm{N}}(\mathbf{a}) \wedge \operatorname{Mon}_{F}^{\mathrm{N}}(\mathbf{a}) \rightarrow(\exists x \subseteq \mathrm{N}) F(\mathbf{a}, x, x) .
$$

Proof. Suppose that $\mathrm{Op}_{F}^{\mathrm{N}}(\mathbf{a})$ and $\operatorname{Mon}_{F}^{\mathrm{N}}(\mathbf{a})$ hold. Next we set

$$
D(\mathbf{u}, x, y, z):=\left\{\begin{array}{l}
(\bigcup \operatorname{Rng}(y) \nsubseteq \mathrm{N} \wedge z=\bigcup \operatorname{Rng}(y)) \vee \\
(\bigcup \operatorname{Rng}(y) \subseteq \mathrm{N} \wedge x=x \wedge F(\mathbf{u}, \bigcup \operatorname{Rng}(y), z)) .
\end{array}\right.
$$

Now assume that $\operatorname{Wo}(b, c)$. Since $\operatorname{Op}_{F}^{\mathrm{N}}(\mathbf{a})$ and $\operatorname{Mon}_{F}^{\mathrm{N}}(\mathbf{a})$ it follows immediately from the definition of $D$ that

$$
(\forall x \in b) \forall y \exists ! z D(\mathbf{a}, x, y, z) .
$$

Hence Theorem 1 yields that there is a hierarchy $f$ such that $\operatorname{Hier}_{D}(\mathbf{a}, b, c, f)$ holds. Moreover we can prove that $f$ has the following properties expressed by the $\Sigma$ formula $B(\mathbf{a}, b, c, f)$ that is the conjunction of the three formulas below:

(1) $(\exists x \in b)(\forall y \in b)((y, x) \notin c)$,

(2) $(\forall x, y \in b)[(x, y) \in c \rightarrow f(x) \subseteq f(y) \subseteq \mathrm{N}]$,

(3) $(\forall x \in b)(\forall n \in \mathrm{N})[n \in f(x) \rightarrow(\exists y \in b)(x=y \vee(y, x) \in c) \wedge(n \in f(y) \wedge$ $(\forall z \in b)(z, y) \in c \rightarrow(n \notin f(z)))]$.

Thus $B(\mathbf{a}, b, c, f)$ states that the set $b$ has a least element with respect to the ordering $c$, that the function $f$ is monotone with $\operatorname{Rng}(f) \subseteq \mathrm{P}(\mathrm{N})$ and that if $n$ is an element of $f(x)$ for some $x \in b$, then there is a least $y$ with respect to the ordering $c$ such that $n \in f(y)$.

The first and the third property follow directly from that fact that $c$ is a wellordering on $b$. The second property is shown by transfinite induction along $c$ on $b$ : We set

$$
C(v):=(\forall x, y \in b)[(x, y) \in c \wedge(y, v) \in c \rightarrow f(x) \subseteq f(y) \subseteq \mathrm{N}]
$$

and assume that $w \in b$ and that for all $v \in b$ with $(v, w) \in c$ we have $C(v)$. We aim to show that $C(w)$. So assume that $x, y \in b$ with $(x, y) \in c$ and $(y, w) \in c$. $\operatorname{Hier}_{D}(\mathbf{a}, b, c, f)$ implies that $D(\mathbf{a}, y,\{(z, f(z)):(z, y) \in c\}, f(y))$. Now $C(y)$ implies that $\bigcup\{f(z):(z, y) \in c\} \subseteq \mathrm{N}$. Hence the definition of $D$ and $\operatorname{Mon}_{F}^{\mathrm{N}}(\mathbf{a})$ yield $f(x) \subseteq \bigcup\{f(z):(z, y) \in c\} \subseteq f(y) \subseteq \mathrm{N}$. But this is $C(w)$.

So we have shown that $\operatorname{Op}_{F}^{\mathrm{N}}(\mathbf{a})$ and $\operatorname{Mon}_{F}^{\mathrm{N}}(\mathbf{a})$ imply

$$
\forall b, c\left[\mathrm{Wo}(b, c) \rightarrow \exists f\left(\operatorname{Hier}_{D}(\mathbf{a}, b, c, f) \wedge B(\mathbf{a}, b, c, f)\right)\right] .
$$

By lemma 5 we conclude that there exist sets $d, e$ and a function $g$ such that

$$
\mathrm{PSH}_{D}(\mathbf{a}, d, e, g) \wedge B(\mathbf{a}, d, e, g) \text {. }
$$

Next, we divide $d$ in an upper part $u$ and a lower part $l$. Because $\neg \operatorname{Wo}(d, e)$ there is a set $u \subseteq d$ that has no least element with respect to the linear ordering $e$. Further we can assume that the set $u$ is upwards closed, i.e. if $x \in u$ and if $(x, y) \in e$ then also 
$y \in u$. The lower part $l \subseteq d$ is now defined as $l:=\{x \in d:(\forall y \in u)(x, y) \in e\}$. Since $d$ has a least element with respect to the ordering $e, l$ is not empty.

Looking for a candidate for a fixed point of the operator $F$, we set

$$
\begin{aligned}
w & :=\bigcup_{x \in l} g(x), \\
w^{\prime} & :=\bigcap_{x \in u} g(x) .
\end{aligned}
$$

The monotonicity of $g$ implies $w \subseteq w^{\prime}$, but also $w^{\prime} \subseteq w$ holds: If $y \in w^{\prime}$ there is a least element $x_{0} \in d$ with respect to the ordering $e$ such that $y \in g\left(x_{0}\right)$. Because $u$ has no least element with respect to the ordering $e, x_{0}$ belongs already to $l$, yielding $y \in w$. This means that $w=w^{\prime}$. Next we argue that $w$ is indeed a fixed point of $F$.

First, we fix a set $z$ such that $F(\mathbf{a}, w, z)$ holds, and then we choose an arbitrary $x \in l$. We have that

$$
F(\mathbf{a}, \bigcup\{g(y):(y, x) \in e\}, g(x)) .
$$

Further, $\operatorname{Mon}_{F}^{\mathrm{N}}(\mathbf{a})$ and the definition of $w$ imply $g(x) \subseteq z$. This means that for all $x \in l$ we have $g(x) \subseteq z$, hence $w \subseteq z$.

Similarly, for an arbitrary $x \in \bar{u}$,

$$
F(\mathbf{a}, \bigcup\{g(y):(y, x) \in e\}, g(x))
$$

holds. This time we conclude that $z \subseteq g(x)$. So for all $x \in u$ we have $z \subseteq g(x)$, hence $z \subseteq w^{\prime}$. But since $w=w^{\prime}$ we have $z=w$ and therefore $F(\mathbf{a}, w, w)$.

This concludes the argument that $\left[\mathrm{KPi}^{0}+(\Sigma-\mathrm{TR})\right]^{\dagger}$ proves the principle $(\Sigma-\mathrm{FP})$. Due to lemma 2 , the theories $\mathrm{KPi}^{0}+(\Sigma-\mathrm{TR})$ and $\left[\mathrm{KPi}^{0}+(\Sigma-\mathrm{TR})\right]^{\dagger}$ have the same proof-theoretic ordinal.

\section{Theorem 3.}

$$
\left|\mathrm{KPi}^{0}+(\Sigma-\mathrm{FP})\right| \leq \varphi \omega 00
$$

\section{Lower bound}

The purpose of this section is to prove that $\varphi \omega 00$ is also a lower bound for the theory $\mathrm{KPi}^{0}+(\Sigma-\mathrm{FP})$. Our strategy is to adapt the well-ordering proof for $\mathrm{KPi}^{0}+(\Sigma-\mathrm{TR})$ given in [6] to the present context. This well-ordering proof is based on the wellordering proof in Jäger, Kahle, Setzer and Strahm [5]. Also Rüede [10] adopts this proof for the treatment of $\Sigma_{1}^{1}$ transfinite dependence choice.

In the well-ordering proof in [6], one defines for each natural number $n$, a $\Delta_{0}$ formula $\mathcal{K}_{n+1}(x)$ of $\mathcal{L}^{*}$ that satisfies the following property:

$$
\mathcal{K}_{n+1}(y) \rightarrow(\forall x \in y) \operatorname{TI}(x, \varphi \hat{n} 00) .
$$

Then one proves the so called Main Lemma, claiming that for every set $x$, there exists a set $y$, such that $x \in y$ and $\mathcal{K}_{n+1}(y)$. As an immediate consequence, the ordinal $\varphi \hat{n} 00$ is provable for every natural number $n$. 
We start by repeating the definitions of the $\Delta_{0}$ formulas $\mathcal{K}_{n}(y)$. They express that $y$ respects certain closure properties regarding hierarchies. So $\mathcal{K}_{0}(a)$ says that $a$ is an admissible, and $\mathcal{K}_{n+1}(a)$ states that $a$ is a limit of admissibles that contains with a set $x$ also a $\mathcal{K}_{n}$-hierarchy $f$ above $x$, whose domain is the set of notations $\mathfrak{a} \in$ OT that look like a well-ordering in $a$. The formal definitions are given below.

\section{Definition 1.}

$$
\begin{aligned}
\overline{\operatorname{Ad}}(a) & :=\operatorname{Ad}(a) \vee(\exists u(u \in a) \wedge(\forall x \in a)(\exists y \in a)(x \in y \wedge \operatorname{Ad}(y))) \\
\mathcal{T}(f) & :=\operatorname{Fun}(f) \wedge \operatorname{Dom}(f)=\mathrm{OT}, \\
\mathcal{K}_{1}(a) & :=\operatorname{Ad}(a), \\
\mathcal{K}_{n+1}(a) & :=\overline{\operatorname{Ad}}(a) \wedge\left[\forall x \exists f\left(\mathcal{T}(f) \wedge \forall \mathfrak{a}\left(\operatorname{Wo}(\mathfrak{a}) \rightarrow \mathcal{H}_{n}(\mathfrak{a}, x, f)\right)\right)\right]^{a}, \\
\mathcal{H}_{n}(\mathfrak{a}, u, f) & :=\mathcal{T}(f) \wedge(\forall \mathfrak{b} \prec \mathfrak{a})\left(f \uparrow \mathfrak{b} \in f(\mathfrak{b}) \wedge u \in f(\mathfrak{b}) \wedge \mathcal{K}_{n}(f(\mathfrak{b}))\right) .
\end{aligned}
$$

The closure properties expressed by the $\Delta_{0}$ formulas $\mathcal{K}_{n}(x)$ are tailored such that the following lemma holds. For a proof see lemma 11 in [6].

Lemma 6. $\mathrm{KPi}^{0}$ proves for each natural number $n$ that

$$
\mathcal{K}_{n+1}(y) \rightarrow(\forall x \in y) \operatorname{TI}(x, \varphi \hat{n} 00) .
$$

An inspection of the proof of the Main Lemma in [6] yields that one needs to iterate $\Sigma$ operations that map elements of the class $\overline{\mathrm{Ad}}$ on elements of $\overline{\mathrm{Ad}}$ along (primitive) recursive well-orderings on N. Since our axiom $(\Sigma-F P)$ only claims the existence of fixed points of monotone $\Sigma$ operators on the powerset of $\mathrm{N}$, we only can iterate $\Sigma$ operations that map subsets of $\mathrm{N}$ to subsets of $\mathrm{N}$ (cf. lemma 9). To adapt the well-ordering proof, we code elements of a suitable subclass of $\overline{\mathrm{Ad}}$ as subsets of natural numbers. Thereto we draw upon the additional assumption that there exists a set for which transfinite induction up to $\varphi \omega 00$ fails. Hence our Main Lemma takes the following form.

Lemma 7 (Main Lemma). For every natural number $n$ greater than 0 we have that $\mathrm{KPi}^{0}+(\Sigma-\mathrm{FP})$ proves:

$$
\exists x \neg \operatorname{TI}(x, \varphi \omega 00) \rightarrow \forall x \exists y\left(x \in y \wedge \mathcal{K}_{n}(y)\right) .
$$

To remove the additional assumption, observe that we have for all natural numbers $n$ :

(1) $\mathrm{KPi}^{0}+(\Sigma-\mathrm{FP}) \vdash \forall x \operatorname{TI}(x, \varphi \omega 00) \rightarrow \forall x \operatorname{TI}(x, \varphi \hat{n} 00)$,

(2) $\mathrm{KPi}^{0}+(\Sigma-\mathrm{FP}) \vdash \exists x \neg \mathrm{TI}(x, \varphi \omega 00) \rightarrow \forall x \mathrm{TI}(x, \varphi \hat{n} 00)$.

(1) is a trivial observation and (2) follows from lemma 6 and lemma 7. Together, they imply that $\mathrm{KPi}^{0}+(\Sigma-\mathrm{FP})$ proves the ordinal $\varphi \hat{n} 00$ for each natural number $n$.

Next we introduce the class $\mathrm{Ad}_{\circ}$ of good admissibles, i.e. admissibles that contain a set for which transfinite induction up to $\varphi \omega 00$ fails,

$$
\operatorname{Ad}_{\circ}(a): \Leftrightarrow \operatorname{Ad}(a) \wedge(\exists x \in a) \neg \operatorname{TI}(x, \varphi \omega 00) .
$$


Of course, good admissibles are also linearly ordered and there is always a next good admissible. It is clear that the existence of good admissibles is an immediate consequence of the assumption $\exists x \neg \mathrm{TI}(x, \varphi \omega 00)$. Below we show how to represent good admissibles as subsets of the natural numbers. For that purpose, we assign to an admissible $a$ the set $\mathrm{p}(a) \subseteq \mathrm{N}$ given by

$$
\left\{e \in \mathrm{N}:(\forall x \in \mathrm{N})\{e\}(x) \in\{0,1\} \wedge \mathrm{Wo}^{a}(\{(m, n) \in \mathrm{N}:\{e\}(\langle m, n\rangle)=0\})\right\} .
$$

So $\mathrm{p}(a)$ is the set of all indices of characteristic functions whose associated relations are well-orderings in $a$. As usual $\{\cdot\}$ denotes Kleene brackets.

Lemma 8. $\mathrm{KPi}^{0}+(\Sigma-\mathrm{FP})$ proves:

$$
\operatorname{Ad}_{\circ}(a) \rightarrow \mathrm{p}(a) \notin a .
$$

Proof. Suppose that $a$ is a good admissible. We argue that $\mathrm{p}(a) \in a$ implies that $a$ is a model of $\Pi_{1}^{1}-\mathrm{CA}_{0}^{-}$, i.e. the theory that extends $\mathrm{ACA}_{0}$ by comprehension for $\Pi_{1}^{1}$ formulas that do not contain set parameters. However this contradicts the definition of $\mathrm{Ad}_{\circ}$, namely that $(\exists x \in a) \neg \mathrm{TI}(x, \varphi \omega 00)$.

Let $\Psi(u, \mathbf{v})$ be a $\Pi_{1}^{1}$ formula of $L_{2}$ without set parameters. As in the proof of lemma $4, \mathrm{ACA}_{0}$ proves that for all natural numbers $\mathbf{m}$ there is a set $T$ dependent on $\Psi$ and the parameters $\mathbf{m}$ such that for all $x$ :

(1) $(T)_{x}$ is a tree,

(2) $(T)_{x}$ is recursive,

(3) $\Psi(x, \mathbf{m}) \leftrightarrow \mathrm{WO}\left(\operatorname{KB}\left((T)_{x}\right)\right)$.

Since $a$ is a model of $\mathrm{ACA}_{0}, \mathrm{KPi}^{0}+(\Sigma-\mathrm{FP})$ proves that

$$
(\forall x \in \mathrm{N})\left[\left(\Psi^{*}\right)^{a}(x, \mathbf{m}) \leftrightarrow \mathrm{Wo}^{a}\left(\left\{(u, v):\langle u, v\rangle \in \mathrm{KB}\left((T)_{x}\right)\right\}\right)\right] .
$$

On the other hand, the definition of $\mathrm{p}(a)$ yields that for any recursive ordering $S \subseteq N$, we have that $\mathrm{Wo}^{a}(\{(u, v):\langle u, v\rangle \in S\})$ is equivalent to

$$
(\exists e \in \mathrm{p}(a))(\forall u, v \in \mathrm{N})[\{e\}(\langle u, v\rangle)=1 \leftrightarrow\langle u, v\rangle \in S] .
$$

For all $x \in \mathrm{N}$ the set $\mathrm{KB}\left((T)_{x}\right)$ is recursive. Since $a$ is also a model of ( $\Delta_{0}$-Sep) and $\mathrm{p}(a) \in a$, there exists a set $Y \in a$ such that

$$
\forall x\left[x \in Y \leftrightarrow x \in \mathrm{N} \wedge\left(\Psi^{*}\right)^{a}(x, \mathbf{m})\right] .
$$

This means that $a$ is a model of $\Pi_{1}^{1}-\mathrm{CA}_{0}^{-}$.

So if $a$ is a good admissible, $\mathrm{p}(a) \notin a$ holds. Since $\mathrm{p}(a) \in a^{+}$, the linearity of admissibles yields that $\mathrm{p}(a)^{+}=a^{+}$. This allows us to regain the admissible $a$ from the set $\mathrm{p}(a)$. We define

$$
\mathrm{q}(x):= \begin{cases}\emptyset^{+} & : x \in \emptyset^{+} \\ \text {the unique } y \text { with } \operatorname{Ad}(y) \wedge y^{+}=x^{+} & : \text {otherwise }\end{cases}
$$

and obtain the following corollary. 
Corollary 1. The following is provable in $\mathrm{KPi}^{0}+(\Sigma-\mathrm{FP})$ :

$$
\operatorname{Ad}_{\circ}(a) \rightarrow a=\mathrm{q}(\mathrm{p}(a)) .
$$

Next we show that $\mathrm{KPi}^{0}+(\Sigma-\mathrm{FP})$ enables us to iterate $\Sigma$ operations that map subsets of natural numbers to subsets of natural numbers along well-orderings on subsets of N. First we introduce some auxiliary notions.

$$
\begin{aligned}
\operatorname{Fun}_{\mathrm{N}}(f) & :=f \subseteq \mathrm{N} \wedge(\forall x \in f)(\exists y, z)(x=\langle y, z\rangle), \\
\text { Support }_{\mathrm{N}}(f) & :=\{x \in \mathrm{N}:(\exists y \in \mathrm{N})(\langle x, y\rangle \in f)\} .
\end{aligned}
$$

Fun $_{\mathrm{N}}(f)$ expresses that $f$ is a subset of $\mathrm{N}$ consisting of codes of ordered pairs. Such an $f$ can be seen to represent a function $g: \mathrm{N} \rightarrow \mathrm{P}(\mathrm{N})$,

$$
g:=\{(x, y): x \in \mathrm{N} \wedge y=\{n \in \mathrm{N}:\langle x, n\rangle \in f\}\} .
$$

If $n \notin$ Support $_{\mathrm{N}}(f)$ then $g(n)=\emptyset$. Further, we say that $f$ is an $\mathrm{N}$-hierarchy for the $\mathcal{L}^{*}$ formula $D(\mathbf{u}, x, y, z)$ with parameters a along the well-ordering $c$ on $b$, if $\operatorname{Hier}_{D}^{\mathrm{N}}(\mathbf{a}, b, c, f)$, where $\operatorname{Hier}_{D}^{\mathrm{N}}(\mathbf{a}, b, c, f)$ is the formula

$$
\begin{aligned}
& \operatorname{Fun}_{\mathrm{N}}(f) \wedge \operatorname{Support}_{\mathrm{N}}(f) \subseteq b \wedge \\
& (\forall x \in b) D(\mathbf{a}, x,\{\langle v, w\rangle \in f:(v, x) \in c\},\{w:\langle x, w\rangle \in f\}) .
\end{aligned}
$$

Lemma 9. For all $\Sigma$ formulas $D(\mathbf{u}, x, y, z)$ of $\mathcal{L}^{*}, \mathrm{KPi}{ }^{0}+(\Sigma-\mathrm{FP})$ proves: If $b \subseteq \mathrm{N}$ and $\mathrm{Wo}(b, c)$ then

$$
(\forall x \in b)(\forall y \subseteq \mathrm{N})(\exists ! z \subseteq \mathrm{N}) D(\mathbf{a}, x, y, z) \rightarrow \exists f \operatorname{Hier}_{D}^{\mathrm{N}}(\mathbf{a}, b, c, f) .
$$

Proof. We adapt Avigad's argument from [1], which shows that $\mathrm{ATR}_{0}$ proves the existence of fixed points of monotone operators given by an arithmetical formula, to the present context.

Assume that we have sets $\mathbf{a}, b \subseteq \mathrm{N}$ and $c$ satisfying the left hand side of the implication. We aim to find an $f \subseteq \overline{\mathrm{N}}$ such that $\operatorname{Hier}_{D}^{\mathrm{N}}(\mathbf{a}, b, c, f)$. Thereto, a monotone operator is defined, whose fixed point $g \subseteq \mathrm{N}$ codes the characteristic function of $f$.

First, we introduce two auxiliary operations mapping $P(N) \times P(N) \rightarrow P(N)$, monotone in each component. Thereby, if $x \subseteq \mathrm{N}$, we write $\langle x\rangle$ for the set $\{\langle m, n\rangle$ : $\langle m, n\rangle \in x\}$ and $\bar{x}$ for the set $\{n \in \mathrm{N}: n \notin x\}$.

$$
\begin{aligned}
& B_{1}(\mathbf{u}, w, x, y, z):=\left\{\begin{array}{l}
(z=\mathrm{N} \wedge\langle x\rangle \cup\langle y\rangle=\langle\mathrm{N}\rangle \wedge\langle x\rangle \cap\langle y\rangle \neq \emptyset) \vee \\
(D(\mathbf{u}, w, x, z) \wedge\langle x\rangle \cup\langle y\rangle=\langle\mathrm{N}\rangle \wedge\langle x\rangle \cap\langle y\rangle=\emptyset) \vee \\
(z=\emptyset \wedge\langle x\rangle \cup\langle y\rangle \neq\langle\mathrm{N}\rangle),
\end{array}\right. \\
& B_{2}(\mathbf{u}, w, x, y, z):=\left\{\begin{array}{l}
(z=\mathrm{N} \wedge\langle x\rangle \cup\langle y\rangle=\langle\mathrm{N}\rangle \wedge\langle x\rangle \cap\langle y\rangle \neq \emptyset) \vee \\
(D(\mathbf{u}, w, x, \bar{z}) \wedge\langle x\rangle \cup\langle y\rangle=\langle\mathrm{N}\rangle \wedge\langle x\rangle \cap\langle y\rangle=\emptyset) \vee \\
(z=\emptyset \wedge\langle x\rangle \cup\langle y\rangle \neq\langle\mathrm{N}\rangle) .
\end{array}\right.
\end{aligned}
$$

So if $x$ contains exactly those pairs not in $y$ and the operation defined by $D$ assigns $z$ to $x$, then the operations given by $B_{1}$ and $B_{2}$ assign to $x$ and $y$ the sets $z$ and 
$\bar{z}$, respectively. The other cases are defined to assure monotonicity in both components.

For $s \subseteq \mathrm{N}$ and $w \in b$, we further set

$$
\begin{aligned}
& \operatorname{lev}(s, w, c):=\{\langle v, n\rangle:\langle\langle v, n\rangle, 0\rangle \in s \wedge(v, w) \in c\}, \\
& \overline{\operatorname{lev}}(s, w, c):=\{\langle v, n\rangle:\langle\langle v, n\rangle, 1\rangle \in s \vee(v, w) \notin c\} .
\end{aligned}
$$

Under the assumption that $s$ codes the characteristic function of a hierarchy and $w \in b$, then $\operatorname{lev}(s, w, c)$ is the disjoint union of all levels below $w$, and $\overline{\operatorname{lev}}(s, w, c)$ is its complement w.r.t. pairs. Now we choose $C(\mathbf{u}, v, w, x, y)$ to be a $\Sigma$ formula expressing that $y$ is the union of the two sets

$$
\begin{aligned}
& \left\{\langle\langle v, n\rangle, 0\rangle: v \in b \wedge n \in \mathrm{N} \wedge \exists z\left[n \in z \wedge B_{1}(\mathbf{a}, v, \operatorname{lev}(x, v, c), \overline{\operatorname{lev}}(x, v, c), z)\right]\right\} \\
& \left\{\langle\langle v, n\rangle, 1\rangle: v \in b \wedge n \in \mathrm{N} \wedge \exists z\left[n \in z \wedge B_{2}(\mathbf{a}, v, \operatorname{lev}(x, v, c), \overline{\operatorname{lev}}(x, v, c), z)\right]\right\}
\end{aligned}
$$

It is not hard to see that $\operatorname{Op}_{C}^{\mathrm{N}}(\mathbf{a}, b, c)$ and $\operatorname{Mon}_{C}^{\mathrm{N}}(\mathbf{a}, b, c)$, which yields a $g$ with $C(\mathbf{a}, b, c, g, g)$. By transfinite induction we show that for all $v \in b$,

$$
(\forall n \in \mathrm{N})(\langle\langle v, n\rangle, 0\rangle \in g \leftrightarrow\langle\langle v, n\rangle, 1\rangle \notin g) .
$$

Obviously, if 0 is the $c$-least element of $b$, then $\operatorname{lev}(g, 0, c)=\emptyset$ and $\overline{\operatorname{lev}}(g, 0, c)=$ $\langle\mathrm{N}\rangle$. Thus the claim follows from the definition of $C$. And if $(*)$ holds for all $v^{\prime} \in b$ with $\left(v^{\prime}, v\right) \in c$, then $\operatorname{lev}(g, v, c) \cup \overline{\operatorname{lev}}(g, v, c)=\langle\mathrm{N}\rangle$ and $\operatorname{lev}(g, v, c) \cap$ $\overline{\operatorname{lev}}(g, v, c)=\emptyset$, which yields the induction step.

Thus, if we let $f:=\{\langle v, n\rangle:\langle\langle v, n\rangle, 0\rangle \in g\}$ and $v \in b$, then the set $z:=\{n:$ $\langle v, n\rangle \in f\}$ satisfies $D(\mathbf{a}, v, \operatorname{lev}(g, v, c), z)$, therefore $\operatorname{Hier}_{D}^{\mathrm{N}}(\mathbf{a}, b, c, f)$.

Next we show that we can iterate $\Sigma$ operations, whose range consists of good admissibles only, along well-orderings on subsets of $\mathrm{N}$.

Lemma 10. If $D(\mathbf{u}, x, y, z)$ is a $\Sigma$ formula of $\mathcal{L}^{*}$, then $\mathrm{KPi}^{0}+(\Sigma-\mathrm{FP})$ proves that the following two assertions

(1) $b \subseteq \mathrm{N} \wedge \mathrm{Wo}(b, c) \wedge(\forall x \in b) \forall y \exists ! z D(\mathbf{a}, x, y, z)$,

(2) $(\forall x \in b) \forall y, z D(\mathbf{a}, x, y, z) \rightarrow \operatorname{Ad}_{\circ}(z)$,

imply

(3) $\exists f \operatorname{Hier}_{D}(\mathbf{a}, b, c, f)$.

Proof. We assume that (1) and (2) hold, and set

$$
u^{*}:=\left\{(x, z): x \in \operatorname{Support}_{\mathrm{N}}(u) \wedge z=\mathrm{q}(\{y:\langle x, y\rangle \in u\})\right\} .
$$

The idea behind this definitions is the following: If $h \subseteq \mathrm{N}$ and $\operatorname{Fun}_{\mathrm{N}}(h)$, then $h^{*}$ is a function that maps elements of $\operatorname{Support}_{\mathrm{N}}(h)$ to admissibles. Now let

$$
D^{\prime}(\mathbf{u}, x, y, z):=\exists r\left(D\left(\mathbf{u}, x, y^{*}, r\right) \wedge z=\mathrm{p}(r)\right) .
$$


By lemma 9 there exists a $g \subseteq \mathrm{N}$ such that $\operatorname{Hier}_{D^{\prime}}^{\mathrm{N}}(\mathbf{a}, b, c, g)$. Then the function

$$
f:=\{(x, y): x \in b \wedge y=\{z:\langle x, z\rangle \in g\}\} .
$$

satisfies $\operatorname{Hier}_{D}(\mathbf{a}, b, c, f)$ : That $\operatorname{Fun}(f)$ and $\operatorname{Dom}(f)=b$ is obvious. It remains to show that

$$
(\forall x \in b) D(\mathbf{a}, x,\{(y, f(y)):(y, x) \in c\}, f(x)) .
$$

We fix an $x \in b$. $\operatorname{Hier}_{D^{\prime}}^{\mathrm{N}}(\mathbf{a}, b, c, g)$ implies that

$$
D^{\prime}(\mathbf{a}, x,\{\langle v, w\rangle:(v, x) \in c\},\{w:\langle x, w\rangle \in g\}) .
$$

By the definition of $D^{\prime}$ there is a set $r$ such that

$$
D\left(\mathbf{a}, x,\{\langle v, w\rangle:(v, x) \in c\}^{*}, r\right) \wedge\{w:\langle x, w\rangle \in g\}=\mathrm{p}(r) .
$$

The definition of $f$ implies that $f(x)=\mathrm{q}(\{w:\langle x, w\rangle \in g\})=r$ and that

$$
\{\langle v, w\rangle:(v, x) \in c\}^{*}=\{(y, f(y)):(y, x) \in c\} .
$$

Therefore, we have for all $x \in b$,

$$
D(\mathbf{a}, x,\{(y, f(y)):(y, x) \in c\}, f(x)),
$$

which is $\operatorname{Hier}_{D}(\mathbf{a}, b, c, f)$.

In his well-ordering proof for second order arithmetic with $\Sigma_{1}^{1}$ transfinite dependent choice, Rüede makes use of $\Pi_{2}^{1}$ reflection on $\omega$-models of $A C A_{0}$ to prove the Main Lemma. In our present context, this part is taken over by $\Pi_{2}$ reflection on a suitable subclass of $\overline{\mathrm{Ad}}$. This suitable subclass of $\overline{\mathrm{Ad}}$ comprises those elements of $\overline{\mathrm{Ad}}$ that are the limits of increasing $\omega$-sequences of good admissibles,

$$
\operatorname{Seq}_{A_{\circ}}(f):=\left\{\begin{array}{l}
\operatorname{Fun}(f) \wedge \operatorname{Dom}(f)=\mathrm{N} \wedge \\
(\forall n \in \mathrm{N})\left[\operatorname{Ad}_{\circ}(f(n)) \wedge f(n) \in f(n+1)\right] .
\end{array}\right.
$$

Thus a sequence $f$ in $\operatorname{Seq}_{\mathrm{Ad}_{\circ}}$ represents the limit $\bigcup \operatorname{Rng}(f)$, which in turn is coded by the following set of natural numbers, $\{\langle n, x\rangle: x \in \mathrm{p}(f(n))\}$. It is not hard to obtain the following generalization of the previous lemma.

Lemma 11. If $D(\mathbf{u}, x, y, z)$ is a $\Sigma$ formula of $\mathcal{L}^{*}$, then $\mathrm{KPi}^{0}+(\Sigma-\mathrm{FP})$ proves that the following two assertions

(1) $b \subseteq \mathrm{N} \wedge \mathrm{Wo}(b, c) \wedge(\forall x \in b) \forall y \exists ! z D(\mathbf{a}, x, y, z)$,

(2) $(\forall x \in b) \forall y, z D(\mathbf{a}, x, y, z) \rightarrow \operatorname{Seq}_{\mathrm{Ad}_{\circ}}(z)$,

imply

(3) $\exists f \operatorname{Hier}_{D}(\mathbf{a}, b, c, f)$.

By means of lemma 10, we get the following uniform variant of $\Pi_{2}$ reflection on $\overline{\mathrm{Ad}}$. 
Lemma $12\left(\Pi_{2}\right.$ reflection on $\left.\overline{\mathrm{Ad}}\right)$. For any $\Sigma$ formula $A(\mathbf{u}, v, w)$ of $\mathcal{L}^{*}$ with at most the displayed variables $\mathbf{u}, v, w$ free, there exists a $\Sigma$ formula $A^{\sharp}(\mathbf{u}, v)$ of $\mathcal{L}^{*}$ with at most the variables $\mathbf{u}, v$ free, so that the following two assertions can be proved in $\mathrm{KPi}^{0}+(\Sigma-\mathrm{FP})+\exists z \neg \mathrm{TI}(z, \varphi \omega 00)$ :

(1) $\forall x \exists y A(\mathbf{a}, x, y) \rightarrow \exists ! f A^{\sharp}(\mathbf{a}, f)$,

(2) $\forall x \exists y A(\mathbf{a}, x, y) \rightarrow$

$$
\forall f\left(A^{\sharp}(\mathbf{a}, f) \rightarrow \operatorname{Seq}_{A d_{\circ}}(f) \wedge \mathbf{a} \in \bigcup \operatorname{Rng}(f) \wedge[\forall x \exists y A(\mathbf{a}, x, y)] \bigcup \operatorname{Rng}(f) .\right.
$$

Proof. Using lemma 10, the proof of lemma 7 in [6] easily translates to the present context.

Now the stage is set to prove the Main Lemma. Actually we show a bit more, so that we can prove the lemma by meta-induction on the natural numbers.

Lemma 13. For every natural number $n$ greater than 0 there exists a $\Sigma$ formula $F_{n}(u, v)$ of $\mathcal{L}^{*}$ such that $\mathrm{KPi}^{0}+(\Sigma-\mathrm{FP})+\neg \mathrm{TI}(x, \varphi \omega 00)$ proves:

(1) $\forall x \exists$ ! $f F_{n}(x, f)$,

(2) $\forall x \forall f\left[F_{n}(x, f) \rightarrow \operatorname{Seq}_{\text {Ad }_{\circ}}(f) \wedge x \in \bigcup \operatorname{Rng}(f) \wedge \mathcal{K}_{n}(\bigcup \operatorname{Rng}(f))\right]$.

Proof. Again, we refer to the proof of the corresponding Main Lemma in [6]. Replace their theorem 6 by our lemma 11.

From the discussion at the beginning of this section we obtain the lower bound.

\section{Theorem 4.}

$$
\left|\mathrm{KPi}^{0}+(\Sigma-\mathrm{FP})\right| \geq \varphi \omega 00 .
$$

Further we can conclude from theorem 3 and results of Jäger and Strahm in [8] that the theories $\mathrm{KPi}^{0}+(\Sigma-\mathrm{TR}), \mathrm{KPi}^{0}+(\Sigma-\mathrm{FP})$ and $\mathrm{KPm}^{0}$ all have the same proof-theoretic ordinal.

\section{Corollary 2.}

$$
\left|\mathrm{KPi}^{0}+(\Sigma-\mathrm{TR})\right|=\left|\mathrm{KPi}^{0}+(\Sigma-\mathrm{FP})\right|=\left|\mathrm{KPm}^{0}\right|=\varphi \omega 00 .
$$

\section{Concluding remarks}

As mentioned earlier, the restriction in the formulation of the axiom ( $\Sigma-\mathrm{FP})$ to operators on the powerset of the natural numbers is to keep the theory persistent. We will elaborate on this point a bit further.

This time, we set for all formulas $A(\mathbf{u}, v, w)$ of $\mathcal{L}^{*}$,

$$
\begin{aligned}
\mathrm{Op}_{A}(\mathbf{a}) & :=\forall x \exists ! y[\mathrm{~S}(x) \rightarrow \mathrm{S}(y) \wedge A(\mathbf{a}, x, y)], \\
\operatorname{Mon}_{A}(\mathbf{a}) & :=\forall u, v, x, y[A(\mathbf{a}, u, x) \wedge A(\mathbf{a}, v, y) \wedge u \subseteq v \rightarrow x \subseteq y] .
\end{aligned}
$$

The principle $\left(\Sigma-\mathrm{FP}^{\prime}\right)$, claiming the existence of fixed points of monotone $\Sigma$ operators on the whole universe, takes now the following form: For each $\Sigma$ formula $A(\mathbf{u}, v, w)$ of $\mathcal{L}^{*}$ with at most the variables $\mathbf{u}, v, w$ free, we have

$$
\mathrm{Op}_{A}(\mathbf{a}) \wedge \operatorname{Mon}_{A}(\mathbf{a}) \rightarrow \exists x[\mathrm{~S}(x) \wedge A(\mathbf{a}, x, x)] .
$$


From the proof of theorem 2 it follows that $\left[\mathrm{KPi}^{0}+(\Sigma-\mathrm{TR})\right]^{\dagger}$ proves also all instances of the principle $\left(\Sigma-\mathrm{FP}^{\prime}\right)$. However, if we further strengthen the theory $\mathrm{KPi}^{0}+\left(\Sigma-\mathrm{FP}^{\prime}\right)$ by an axiom claiming foundation

$$
\forall x(\exists u(u \in x) \rightarrow(\exists y \in x)(y \cap x=\emptyset)),
$$

(Foundation)

it becomes inconsistent.

To see this, we consider the operation

$$
\mathrm{o}(x):=\bigcup\{\alpha+1: \alpha \in x \wedge \operatorname{Ord}(\alpha)\}
$$

Note that $\mathrm{O}(x)$ is an ordinal and that $\mathrm{O}(\alpha)=\alpha$ for all ordinals $\alpha$. The theory $\mathrm{KPi}^{0}$ also proves that ordinals are linearly ordered by the elementhood relation $\in$.

Lemma 14. $\mathrm{KPi}^{0}$ proves that the operation $x \mapsto \mathrm{O}(x) \cup\{\mathrm{O}(x)\}$ is monotone but has no fixed point.

Proof. Ordinals are linarly ordered by the $\in$ relation, hence the operation is monotone. Since ordinals are well-founded by $\in$, it has no fixed point.

In the presence of foundation, being an ordinal is a $\Delta$ predicate, i.e. $\operatorname{Ord}(\alpha)$ is equivalent to $\operatorname{Tran}(\alpha) \wedge(\forall x \in \alpha) \operatorname{Tran}(x)$. Thus, the aforementioned operation becomes definable by a $\Sigma$ formula of $\mathcal{L}^{*}$, which implies the inconsistency of $\mathrm{KPi}^{0}+$ $\left(\Sigma-\mathrm{FP}^{\prime}\right)+$ (Foundation). Moreover, the operation $x \mapsto \mathrm{O}(x) \cup\{\mathrm{O}(x)\}$ is definable by a $\Sigma$ formula of $\mathcal{L}^{*}$ in any extension of $\mathrm{KPi}^{0}$ where $\mathrm{Wo}(u, v)$ is a $\Delta$ formula, i.e. where there exists a $\Sigma$ formula $A(\mathbf{u}, v, w)$ of $\mathcal{L}^{*}$ such that

$$
\exists \mathbf{x} \forall y, z[\operatorname{Wo}(y, z) \leftrightarrow A(\mathbf{x}, y, z)]
$$

is provable. Due to the previous lemma, such an extension cannot consistently be further extended by the principle $\left(\Sigma-\mathrm{FP}^{\prime}\right)$.

We conclude this section by relating the consistency of $\mathrm{T}+\left(\Sigma-\mathrm{FP}^{\prime}\right)$ to the consistency of $\mathrm{T}+(\Sigma-\mathrm{TR})$ for theories $\mathrm{T}$ comprising $\mathrm{KPi}^{0}$.

Lemma 15. There is a $\Sigma$ formula $A(u, v)$ of $\mathcal{L}^{*}$ such that $\mathrm{KPi}^{0}$ proves $\mathrm{Op}_{A}, \mathrm{Mon}_{A}$ and

$$
A(x, y) \rightarrow y=\bigcap\{z: x \subseteq z \wedge \operatorname{Ad}(z)\}
$$

This justifies the notation $x^{\circ}$ for the set $\bigcap\{z: x \subseteq z \wedge \operatorname{Ad}(z)\}$.

Proof. There is a $\Sigma$ formula $A(u, v)$ of $\mathcal{L}^{*}$ such that $A(x, y)$ implies that

$$
y=\bigcap\left\{z \in\left(x^{+}\right)^{+}: x \subseteq z \wedge \operatorname{Ad}(z)\right\} .
$$

Since admissibles are linearly ordered by $\in$, an admissible $z$ that is not an element of $\left(x^{+}\right)^{+}$satisfies already $x \subseteq x^{+} \subseteq z$. Thus, $A(x, y)$ implies $y=x^{\circ} . \mathrm{Op}_{A}$ and Mon $_{A}$ are now obvious. 
Lemma 16. $\mathrm{KPi}^{0}+\left(\Sigma-\mathrm{FP}^{\prime}\right)$ proves each instance of $(\Sigma-\mathrm{TR})$.

Proof. Suppose that $A(\mathbf{u}, v, w)$ is a $\Sigma$ formula of $\mathcal{L}^{*}$ and $\mathbf{a}$ are sets, such that $\mathrm{Op}_{A}(\mathbf{a})$ and $\operatorname{Mon}_{A}(\mathbf{a})$ hold. Further, assume $\operatorname{Ord}(b)$. We show that $\exists f \operatorname{Hier}_{A}(\mathbf{a}, b, f)$.

By the previous lemma there is a $\Sigma$ formula $B$ with $\mathrm{Op}_{B}(\mathbf{a}, b)$ and $\operatorname{Mon}_{B}(\mathbf{a}, b)$, such that $B(\mathbf{a}, b, x, y)$ implies

$$
y=(\{v: A(\mathbf{a}, u, v) \wedge u \in x\} \cup\{b\})^{\circ} .
$$

Now $\left(\Sigma\right.$ - $\left.\mathrm{FP}^{\prime}\right)$ yields a set $z$ satisfying $B(\mathbf{a}, b, z, z)$. By transfinite induction along $b$ we show that $(\forall w \in b)(\exists ! f \in z) \operatorname{Hier}_{A}(\mathbf{a}, w, f)$. So suppose that $w \in b$ and for all $v \in w$, there is exactly one $g \in z$ satisfying $\operatorname{Hier}_{A}(\mathbf{a}, v, g)$. By $\Sigma$ replacement, there is a unique function $h$ with domain $w$, such that for $v \in w, h(v) \in z$ and $\operatorname{Hier}_{A}(\mathbf{a}, v, h(v))$. Moreover, $h$ is an element of each admissible that is a superset of $z$, thus $h \in z$. For the induction step, let $s:=\left\{\left(v^{\prime}, c\right) \in h(v): v^{\prime} \in v \in w\right\}$ and $f$ the set with $A(\mathbf{a}, s, f)$. This yields $\operatorname{Hier}_{A}(\mathbf{a}, w, f)$. Similarly, we obtain $\exists f \operatorname{Hier}_{A}(\mathbf{a}, b, f)$.

Hence one can consistently extend a theory $\mathrm{T}$ by the principle $\left(\Sigma-\mathrm{FP}^{\prime}\right)$ if and only if it is consistent to assume that $\mathrm{Wo}(u, v)$ is not a $\Delta$ formula of $\mathrm{T}+(\Sigma-\mathrm{TR})$.

Theorem 5. Let $\mathrm{T}$ be a theory that comprises $\mathrm{KPi}^{0}$. Then $\mathrm{T}+\left(\Sigma-\mathrm{FP}^{\prime}\right)$ is consistent if and only if there is no $\Sigma$ formula $A(\mathbf{u}, v, w)$ of $\mathcal{L}^{*}$ for which $\mathrm{T}+(\Sigma$-TR) proves $\exists \mathbf{x} \forall y, z[A(\mathbf{x}, y, z) \leftrightarrow \operatorname{Wo}(y, z)]$.

Proof. Suppose that $\mathrm{T}+\left(\Sigma-\mathrm{FP}^{\prime}\right)$ is consistent. Lemma 16 yields $(\Sigma-\mathrm{TR})$. If Wo were a $\Delta$ predicate, the operation of lemma 14 would be definable by a $\Sigma$ formula of $\mathcal{L}^{*}$, which contradicts $\left(\Sigma-\mathrm{FP}^{\prime}\right)$.

If we assume the right hand side, we can consistently extend $T+(\Sigma-T R)$ by a principle that claims that we have for every $\Sigma$ formula $A(\mathbf{u}, v, w)$ of $\mathcal{L}^{*}$ that

$$
\forall \mathbf{x} \neg(\forall y, z[A(\mathbf{x}, y, z) \leftrightarrow \mathrm{Wo}(y, z)]) .
$$

Now we can use the proof of theorem 2 to show $\left(\Sigma-\mathrm{FP}^{\prime}\right)$.

\section{References}

1. Avigad, J.: On the relationship between $A T R_{0}$ and $\widehat{I D}_{<\omega}$. The Journal of Symbolic Logic 61 (3), 768-779 (1996)

2. Barwise, J.: Admissible sets and structures: An approach to definability theory. Springer, Berlin, 1975

3. Jäger, G.: The strength of admissibility without foundation. The Journal of Symbolic Logic 49 (3), 867-879 (1984)

4. Jäger, G.: Theories for admissible sets: A unifying approach to proof theory. Bibliopolis, Napoli, 1986

5. Jäger, G., Kahle, R., Setzer, A., Strahm, T.: The proof-theoretic analysis of transfinitely iterated fixed point theories. Journal of Symbolic Logic 64 (1), 53-67 (1999)

6. Jäger, G., Probst, D.: Iterating $\Sigma$ operations in admissible set theory without foundation: a further aspect of metapredicative Mahlo. One Hundred Years of Russell's Paradox. (Godehard Link, ed.), de Gruyter, Berlin, 2004, pp. 119-134 
7. Jäger, G., Probst, D.: Variation on a theme of Schütte. Mathematical Logic Quarterly 50 (3), 258-264 (2004)

8. Jäger, G., Strahm, T.: Upper bounds for metapredicative Mahlo in explicit mathematics and admissible set theory. The Journal of Symbolic Logic 66 (2), 935-958 (2001)

9. Pohlers, W.: Proof theory: An introduction, Lecture Notes in Mathematics, Vol. 1407, Springer, Berlin, 1989

10. Rüede, C.: The proof-theoretic analysis of $\Sigma_{1}^{1}$ transfinite dependent choice. Annals of Pure and Applied Logic 121 (1), 195-234 (2003)

11. Schütte, K.: Proof theory, Springer, Berlin, 1977

12. Simpson, S.G.: Subsystems of Second Order Arithmetic. Perspectives in Mathematical Logic, Springer-Verlag, 1998

13. Strahm, T.: Wellordering proofs for metapredicative Mahlo, The Journal of Symbolic Logic 67 (1), 260-278 (2002) 